\title{
Space Telescope
}

\section{Prospects bright on ground}

\section{Baltimore}

THE well-publicized setbacks in the management and construction of the Space Telescope (see Nature 2 June, p.366) seem to have left few scars on those working at the Space Telescope Institute, whose new building will be officially opened this week. Sited on the bank of a meandering stream in a leafy corner of the Johns Hopkins University Baltimore campus, the calm interior of the already filled building seems consistent with the view of the institute's senior staff that their problems have been relatively minor, and will not contribute significantly to delay or extra costs experienced by the Space Telescope mission.

The main problems that face the Space Telescope have arisen principally within Perkin-Elmer, the subcontractor responsible for much of the hardware. The much lauded 2.4-m mirror constructed by Perkin-Elmer has become contaminated within the company's "clean room", significantly decreasing its reflectivity. Although no specific plans have been agreed, everyone expects that the mirror will be cleaned before launch. Another significant problem concerns the fine guidance sensors, interferometric devices giving the telescope the superb positional accuracy which its refined optics require. Problems in the development of these devices at Perkin-Elmer seem likely to delay the project by a year (to late in 1986) and to add $\$ 100$ million to the total cost.

Neither of these problems is the responsibility of the Space Telescope Institute, whose staff expects that the performance of the Space Telescope should be undiminished. Broadly speaking, the job of the institute's director, Riccardo Giacconi, and his staff will be to maintain and schedule the telescope's operation, to coordinate the retrieval and storage of the data from the telescope's six detectors and to administer access by astronomers.

One task nearing completion is the development of the Guide Star Selection System (GSSS). The Fine Guidance System has to scan over the 69 arc- $\mathrm{min}^{2}$ field of view to locate previously programmed stars to guide the telescope. It is hoped that the telescope's pointing error will be as small as 0.007 arc-s. To ensure the presence of usable stars for any orientation, a photographic plate library down to visual magnitude $14.5(2,400$ times fainter than can be seen with the naked eye) has been compiled. By next September, it is expected that the institute's two scanning microdensitometers and their associated VAX $11 / 750$ computers will have been programmed so that the guidance star catalogue can be drawn up before launch.

The total remaining computing hardware at the disposal of the Space Telescope is formidable, including six linked VAX computers (two of which are at the NASA
Goddard Space Flight Center (GSFC) through which all communication with the Space Telescope will be channelled) and ten image processing systems for real time support and for data analysis. The "Science Operations Ground System" which is to incorporate these devices has nevertheless been something of a headache for the institute's personnel.

The original specifications were drawn up by GSFC, with scientific advice, and carried out by TRW Systems Inc. The institute has spent more time than anticipated on revising those initial plans. Adrienne Timothy, the institute's project manager, explained that it might have been better if the institute had had a year's more lead time so that the system's development could have been better coordinated.

Similar problems have arisen in the institute's instrument support branch. The detectors fixed to the Space Telescope's focal plane will inrlude a high speed photo-

meter, faint object camera, wide field/planetary camera, faint object spectrograph and high resolution spectrograph, each of which is being developed elsewhere.

None of these problems appears to threaten the programme. Indeed, Adrienne Timothy described them as the teething problems one might expect in a project of this magnitude. Neverthess, she agreed that the solution to these problems had been achieved partly to the detriment of the institute's research staff. This includes $\mathbf{4 0}$ or so astronomers, mostly experienced workers with established reputations who had expected to spend half their time on research and have instead been coping with the project's teething problems. Negotiations are now under way with NASA and subcontractors in the hope that these difficulties can be alleviated. Certainly the institute's official policy is that, by launch time, the staff should spend half their time on research. Thereafter, most of them will have to compete with the rest of the community for observing time, the peer review process being conducted outside the institute. Philip Campbell

\section{UK-Dutch astronomy}

\section{Mauna Kea telescope in view}

CONSTRUCTION has started on the largely British-Dutch 15-metre millimetre-wave telescope on Mauna Kea, Hawaii. The telescope, costing $£ 7$ million, is to be financed 80 per cent by the UK Science and Engineering Research Council and 20 per cent by the Netherlands Organization for the Advancement of Pure Science. The observatory will be at an altitude of about $14,000 \mathrm{ft}$ - well above most of the water vapour that impedes millimetric observations at lower altitudes - and is expected to be completed by 1986 . The Royal Observatory, Edinburgh, already responsible for the UK Infrared Telescope at the same site, will administer the facility, the combined running costs being $£ 1.5$ million a year.

Two factors apart from atmospheric water vapour have hitherto hindered the development of millimetre-wave telescopes. One has been the lack of sufficiently sensitive receivers, but the recent development of very low temperature detectors with elements only one micrometre square has alleviated such problems. The other factor was the accuracy needed in the construction of the paraboloid reflector surface. Mechanical and thermal distortions should not distort the surface beyond a small fraction of the operating wavelength - in this case, 50 micrometres. These problems should be overcome by the lightweight aluminium honeycomb structure and the thermal protection provided by the telescope's enclosure. The latter incorporates a membrane which will reflect most visible and thermal radiation but which will transmit millimetre radiation.

The new telescope is of particular significance because of the poor state of health of millimetre astronomy in the United States, where plans for a 15-metre millimetre-wavelength telescope collapsed last year. Apart from initial proposals for a new design study for an aperture synthesis array (see Nature 5 May, p.7), there are no comparable US facilities in sight.

A hint of the potential benefits came a few weeks ago, when Japanese astronomers reported the detection of a rotating gas cloud surrounding a massive proto-star in Orion. Using the Nobeyama $45-\mathrm{m}$ dish, they observed the system at a wavelength of $6.1 \mathrm{~mm}$ (a spectral wavelength of carbon monosulphide). Dr N. Kaifu and his collaborators reported that the system contained a central proto-star of about 50 solar masses surrounded by a gaseous disk of 200 solar masses, rotating and gradually contracting from a radius of $40,000 \mathrm{AU}$ (just over half a light-year). However, the presence of a strong solar wind would, said Dr Kaifu, probably prevent the formation of planets.

To some extent, the British and Japanese telescopes will be complementary. While the Japanese telescope is much larger in diameter, the accuracy of its surface paraboloid is to within about $0.2 \mathrm{~mm}$, compared with the $1 / 40 \mathrm{~mm}$ for the BritishDutch telescope. Thus the latter will be able to observe with an astronomical resolution of 10 arc-s resolution at only $1 / 3 \mathrm{~mm}$ wavelength. The Japanese may reach comparable resolution, but at longer wavelengths. In 1985, however, they will have a $5 \times 10 \mathrm{~m}$ dish interferometer with which they hope to observe the $\mathrm{CO}$ line $(2.6 \mathrm{~mm}$ wavelength) with an accuracy of 1 arc-s.

Philip Campbell \& Robert Walgate 